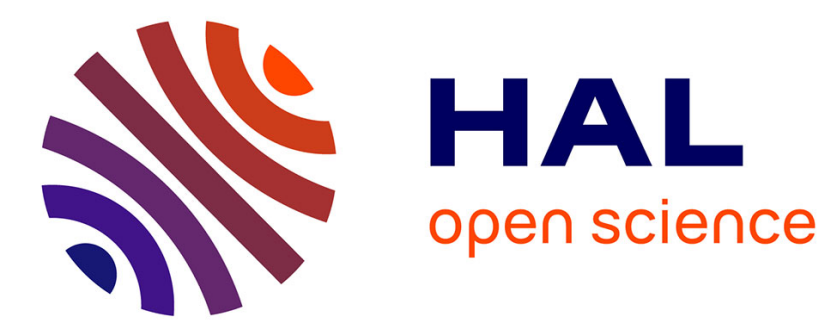

\title{
Gypsum Dissolution Rate from Atomic Step Kinetics
}

Bahareh Zareeipolgardani, Agnès Piednoir, Jean Colombani

\section{To cite this version:}

Bahareh Zareeipolgardani, Agnès Piednoir, Jean Colombani. Gypsum Dissolution Rate from Atomic Step Kinetics. Journal of Physical Chemistry C, 2017, 121 (17), pp.9325. 10.1021/acs.jpcc.7b00612 . hal-01523924

\section{HAL Id: hal-01523924 \\ https://hal.science/hal-01523924}

Submitted on 17 May 2017

HAL is a multi-disciplinary open access archive for the deposit and dissemination of scientific research documents, whether they are published or not. The documents may come from teaching and research institutions in France or abroad, or from public or private research centers.
L'archive ouverte pluridisciplinaire HAL, est destinée au dépôt et à la diffusion de documents scientifiques de niveau recherche, publiés ou non, émanant des établissements d'enseignement et de recherche français ou étrangers, des laboratoires publics ou privés. 


\title{
Gypsum Dissolution Rate from Atomic Step Kinetics
}

\author{
Bahareh Zareeipolgardani, Agnès Piednoir, and Jean Colombani* \\ Institut Lumière Matière; Université de Lyon; Université Claude Bernard Lyon 1; CNRS, \\ UMR 5586; Campus de la Doua, F-69622 Villeurbanne cedex, France \\ E-mail: Jean.Colombani@univ-lyon1.fr
}




\begin{abstract}
The macroscopic dissolution rate of minerals is generally deduced from solution chemistry measurements. A microscopic dissolution rate can also be deduced from the dynamics of molecular events (etch pits growth, atomic step migration ....). Both hardly ever agree, even qualitatively, and the elaboration of a general theory linking the kinetics at the two scales is still in progress. We present here microscopic dissolution rates of gypsum, measured by atomic force microscopy (AFM), in quantitative agreement with macroscopic rates. This agreement has been obtained in taking care to neutralize the bias induced by the force applied by the AFM tip on the surface, and to identify clearly the driving molecular mechanism. This result shows that the determination, among the topographic changes during the dissolution of a mineral, of the dominant one, and the measurement of its dynamics, may permit to deduce from AFM experiments a reliable macroscopic dissolution rate.
\end{abstract}




\section{Introduction}

Beside the measurement of macroscopic reaction rates, the study of the reactivity of minerals includes the investigation of the atomic mechanisms involved in the reaction. This is made possible by the use for two decades of tools resolving nanometric objects, like vertical scanning interferometry (VSI) and atomic force microscopy (AFM). In the case of the study of dissolution, these techniques enable ex-situ as much as in-situ observations, and they have brought recently a rather clear view of this phenomenon. For instance, it is now well accepted that the dissolution kinetics of minerals is driven both by the deepening and broadening of etch pits, and by the general vertical retreat of the surface. ${ }^{1}$ Etch pits are now viewed as contributing to dissolution, not in providing new reactive surface area, but as being source of moving atomic steps. ${ }^{2}$ The way the under-saturation influences dissolution has also received a satisfactory explanation: dissolution in close-to-equilibrium conditions is driven by existing steps, far-from-equilibrium conditions by the nucleation of etch pits on plane surface, and intermediate conditions by the nucleation of etch pits on defects. ${ }^{3,4}$

If calcite has received the most interest in dissolution studies, we are here concerned by the case of gypsum. In the field, the dissolution of gypsum leads to the formation of karst, ${ }^{5}$ is a major source of calcium ion release in drinking water, ${ }^{6}$ and has a significant influence on soil quality and plant growth. ${ }^{7}$ In the domain of mineral materials, gypsum constitutes a minor component of Portland cement, and experiences dissolution during its setting. But it is also the only component of gypsum plaster (or stucco, or alabaster), and its dissolution under stress is responsible for the moist creep of this material. ${ }^{8}$

The main objective of this study is to bridge quantitatively the gap between the atomic and macroscopic dissolution kinetics. Indeed, agreement between measured dissolution rates at these two scales is hardly ever obtained. ${ }^{9}$ Even studies measuring the microscopic (with AFM) and macroscopic (with a flow cell) dissolution rates in one well-defined system do not get similar values. ${ }^{10,11}$ This lack of consistency stems from (i) the fact that the understanding of the molecular mechanisms underlying dissolution is quite recent, as explained above, (ii) 
the difficulty to get reliable macroscopic dissolution rates ( $\mathrm{see}^{12}$ for the case of calcite), and (iii) the fact that the elaboration of a general theory showing how the atomic mechanisms combine into an overall rate is still under way. ${ }^{13,14}$

The macroscopic rate derives from the combination of various sources of matter removal (broadening and coalescence of pits created at point defects, at dislocations, at grain boundaries ...) interacting in a complex manner. ${ }^{15}$ But even if the resulting spectrum of microscopic dissolution rates is large, due to the variety of dynamics of these molecular mechanisms, one of them may dominate and drive mainly the overall rate. In this case the upscaling of the dissolution rate is straightforward, easily deduced from the kinetics of the dominant mechanism.

In the case of gypsum, this upscaling had not yet given values compatible with experimental macroscopic ones because only events that had a minor contribution to the rate (migration of straight steps, etch pits spreading) had been investigated. We show here that the overall rates computed from the kinetics of the dominant microscopic mechanism (rough step migration) agree quantitatively with experimental macroscopic ones.

\section{Experiment}

All experiments have been performed with fresh cleaved surfaces of natural transparent gypsum $\left(\mathrm{CaSO}_{4}, 2 \mathrm{H}_{2} \mathrm{O}\right)$, from the quarry of Mazan (Vaucluse, France). This mineral displays a perfect (010) cleavage plane due to the weak H-bonding between the water molecules. ${ }^{16}$ The undersaturated solutions have been prepared in dissolving calcium sulfate in water up to saturation, then adding ultrapure water (resistivity $\geq 0.18 \mathrm{M} \Omega \cdot \mathrm{m}$ ) until the desired saturation index is obtained. The saturation index has been computed as $\Omega=c / c_{\text {sat }}$, where $c$ is the concentration of dissolved calcium sulfate, and $c_{\text {sat }}=15 \mathrm{mmol} / \mathrm{kg}$ is its value at equilibrium. $^{17}$

A commercial atomic force microscope (MFP3D - Asylum Research - Oxford Instruments) 
has been used for the imaging of gypsum surfaces. In this apparatus, a silicon nitride tip is stuck at the free extremity of a cantilever. When approached close to the surface, the interaction force between the surface and the tip induces a bending of the cantilever, detected by a quadrant detector receiving a laser beam reflected by the cantilever. In the contact mode, used here, a feedback loop modifies the vertical position of the tip in order to keep constant the repulsive force. Then a portion of the surface is scanned line by line, which provides a two-dimensional map of the surface height.

The image in 1a is topographic, i.e., shows directly the height of the surface in the $(x, y)$ plane. But to gain clarity, the presented image in $1 \mathrm{~b}$ shows at each point the error signal, i.e., the difference between the vertical deflection and the set-point, proportional to the desired force, which is equivalent to the derivative of the height with the position. This signal emphasizes the relief of the surface. The images have a $256 \times 256$ resolution and are collected in about $4.5 \mathrm{~min}$ with a $1 \mathrm{~Hz} /$ line frequency.

The dissolution has been studied in situ, thanks to a home-made fluid cell, in which the undersaturated solution is flowing past the mineral surface, with a $0.275 \mathrm{~mL} / \mathrm{min}$ flow rate, during the AFM measurements. The continuous flow leads to the establishment of a steady concentration field above the surface. Despite stationary, the concentration distribution in the flow cell is highly non-uniform and strongly dependent on its geometry. ${ }^{18}$ The way this geometry influences the concentration field and, accordingly, the step velocity, is still quite controversial. Finite element methods modeling have shown that the flow rate, cell geometry, and heterogeneity of the surface, can influence strongly the gypsum step velocity measured locally with the AFM. ${ }^{19}$ But measurements with microelectrodes at different heights from a gypsum dissolving surface for a $1.5 \mathrm{~mL} / \mathrm{min}$ flow rate show the quasi-absence of concentration gradient between the surface and the bulk liquid. ${ }^{20}$

To reduce as much as possible the dispersion of the results and gain accuracy in comparison with already existing results, the measurement of the force applied by the tip on the surface was calibrated (zero value and sensitivity) between each image, in order to com- 
pensate possible drifts and to guarantee the reproducibility of the applied pressure and the rightness of its measured value.

The velocity of steps has been measured in aligning one mono-molecular step with the $y$ scanning direction, and disabling the scan in this directions. Then a kymogram was obtained, the step migration leaving a track in the $(x, t)$ plane ( $t$ the time), and the velocity being the slope of the track ${ }^{21}$.

\section{Results and discussion}

\section{Surface topography}

In a flowing saturated solution, the gypsum cleavage surface appears at rest, with atomic steps moving at velocities lower than $0.03 \mathrm{~nm} / \mathrm{s}$. As soon as the imposed concentration departs from its equilibrium value, coherent motions are observed at the crystal surface, which can be classified in two types, as first observed by Fan \& Teng. ${ }^{21}$

First, rhomboidal etch pits growing in depth and width are continuously observed at the surface. We have not tried to estimate whether they nucleate at structural defects ${ }^{22}$ and whether their nucleation stops close to the saturation. ${ }^{21}$ They are enclosed by straight steps, the displacement of which is responsible for the etch pit growth (see figure 1a). The shape of the pit is reminiscent of the monoclinic Bravais lattice of gypsum, the obtuse angle of $118^{\circ}$ of the monoclinic cell inducing an equivalent angle of the rhomboids. Two facing edges of the pit are longer, because the neighboring edges propagate faster, and vice-versa, these fast steps are shorter because the longer ones go slowlier. The fast steps have been identified as the [100] direction of the lattice, and the slow ones as the [001] direction. ${ }^{21}$ The chemical origin of the kinetic anisotropy between [001] and [100] has been analyzed in detail and derives from the difference of molecular stacking in the two steps. ${ }^{21}$ It should be noted that fast steps are sometimes wrongly identified as [101], whereas these steps are generally not present at gypsum cleavage surface. ${ }^{21}$ We observe that some angles of etch pits 
are sometimes slightly rounded, as in other experiments. ${ }^{23}$

Secondly the surface is regularly swept by very fast and rough steps (see two examples in 1b). These steps have an unidentified and changing direction. Scanning progressively all lattice directions of the (010) plane, they have been denoted [u0w]. ${ }^{21}$ These steps do not belong to any etch pits and Fan \& Teng have suggested that they are generated at the edges of the sample. ${ }^{21}$ Although this should be partly true, molecular simulations of dissolving calcium carbonate, where similar rough steps run on the surface, have shown that they may result from the merging of neighboring pits. ${ }^{13}$ These steps are most probably as unstable as would be [010] steps. ${ }^{21}$ Their very high velocity is a consequence of the large density of kink sites, which are highly reactive, due to their smaller number of bonds with the surface, compared to atoms in straight steps. These rough steps erode and often completely annihilate existing etch pits. For this reason, the pits have generally no time to grow and pits deeper than a few nm are hardly ever observed, whatever the under-saturation.

These topographic transformations of the surface are the molecular consequences of the disequilibrium between the solid and liquid induced by the undersaturation. Therefore, the kinetics of these modifications should, in some form, be linked to the distance from equilibrium. Figure 2 shows the change of the [100] step velocity $v$ with the saturation index $\Omega$, measured in this work, and reported in the literature.

We have to mention that we have omitted to include the value measured by Peruffo et al., because it was two orders of magnitude larger than the others $(v=330 \mathrm{~nm} / \mathrm{s}) .{ }^{24}$ This discrepancy may be probably explained by the fact that these measurements have been carried out during the first seconds of contact of the surface with the solution. Being freshly cleaved and unreacted, the gypsum surface is likely to be, in these experiments, highly energetic and therefore much more reactive than in the other long-term experiments.

If we note an enhancement of the step speed when getting away from the saturation, the exact dependence of $v$ on $\Omega$ would be difficult to draw from the results in 2. Fan \& Teng have suggested, based on their results, that this evolution is compatible with a law deduced 

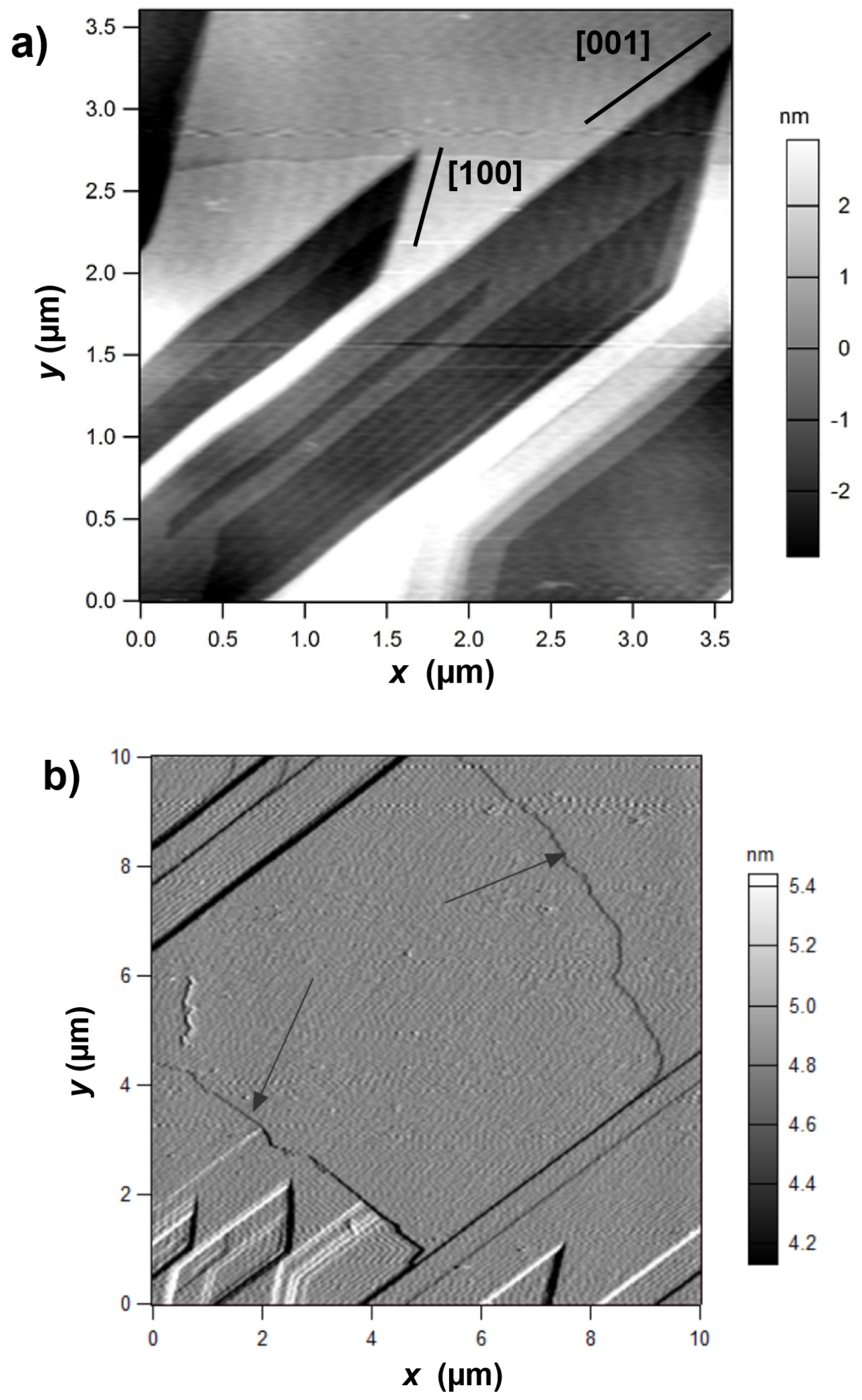

Figure 1: AFM images of the dissolving cleavage surface of a gypsum single crystal in a flowing solution of saturation index $87 \%$. a) Topographic image exhibiting typical rhomboidal etch pits. b) Error signal image showing two fast moving rough steps (arrows). 
from the transition state theory. ${ }^{21}$

At first order, we have considered here that, for our close-to-equilibrium conditions, all measurements converge to a rough proportionality between the step velocity and the distance from saturation, with a dispersion compatible with the difficulty of the experiments (see next section). Therefore, the atomic mechanism at the basis of dissolution seems to follow, close to saturation, a first-order evolution with $\Omega$, as has been observed for its macroscopic counter-part, the dissolution rate $R:^{25}$

$$
v=v_{\lim }(1-\Omega)
$$

with $v_{\text {lim }}$ the step velocity at infinite dilution. Farther from equilibrium, it is likely that the dependency of $v$ with $\Omega$ is no more linear. ${ }^{21}$

\section{Influence of the applied force}

As we aim at a quantitative agreement between atomic and macroscopic dissolution rates, we need to avoid all experimental biases as much as possible. We had shown in a previous work that one of the main instrumental source of uncertainty in AFM dissolution measurements was the modification of the equilibrium between the solid and liquid by the stress $\sigma$ applied by the tip on the surface. ${ }^{26}$ Indeed, the force pressing the surface induces an elastic strain of the solid, which thereby stores the elastic energy

$$
\Delta U_{\mathrm{e}}=\frac{\alpha \sigma^{2} \bar{V}}{2 E}
$$

with $\alpha$ a geometric factor depending on the shape of the elastic strain field around the tip-surface contact, $E$ Young's modulus of the solid, and $\bar{V}$ its molar volume.

This elastic energy constitutes a second source of distance from equilibrium, adding to the undersaturation of the liquid. In other words, the external stress promotes dissolution, a phenomenon broadly reported in geology. ${ }^{27}$ In the case of the dissolution of gypsum, we 


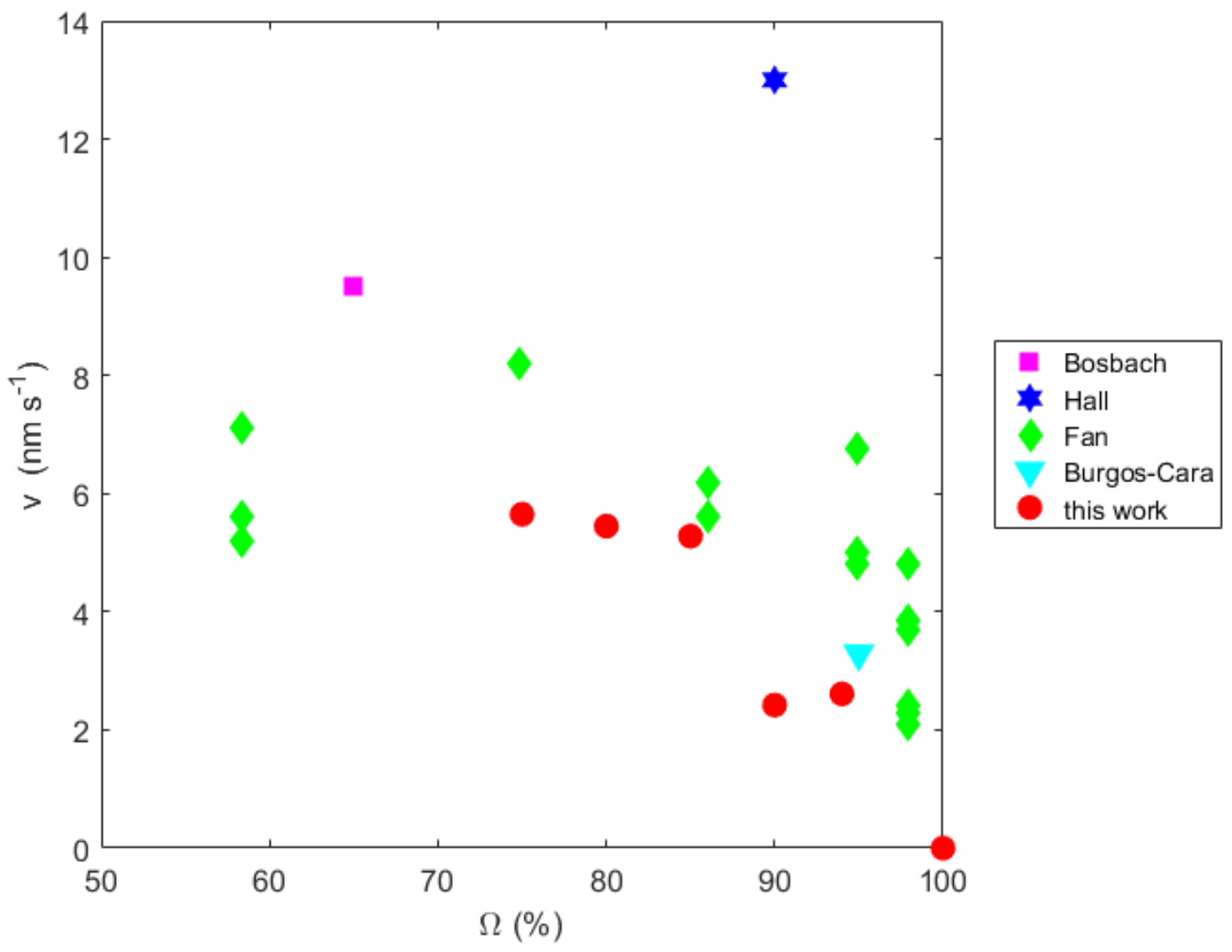

Figure 2: Evolution with the saturation index of the [100] step velocity on a dissolving gypsum surface. The results of Bosbach \& Rammensee (pink square), ${ }^{22}$ Hall \& Cullen (blue hexagon), ${ }^{23}$ Fan \& Teng (green diamonds) ${ }^{21}$ and Burgos-Cara et al. (light blue triangle) ${ }^{20}$ have been added to ours (red circles). 
had demonstrated that the kinetics of the slow steps was thereby modified by the passage of the tip, their dynamics appearing to be driven by a combination of the chemical (1) and mechanical (2) stimuli, both contributing to the Gibbs free energy of the system: ${ }^{26}$

$$
v=v_{\lim }\left[1-\Omega \exp \left(-\frac{\alpha \sigma^{2} \bar{V}}{2 E R T}\right)\right]
$$

To further attest this disturbing influence of the AFM tip on the surface behavior, we present in 3 the change of the velocity of the fast steps with the force applied by the tip, for a given undersaturation (90\%). We see here clearly two distinct regimes. We have ascribed the low force behavior to the above-described pressure solution influence. To use equation 3 , we have modeled the impinging of the surface by the tip with a Hertzian contact. In this case, the maximum tensile stress (at the contact periphery) is $\sigma=((1-2 \nu) / \pi)\left(2 F E^{2} /\left(9\left(1-\nu^{2}\right)^{2} r^{2}\right)\right)$, with $\nu$ Poissons's ratio of the solid, $r$ the size of the contact $(r \sim 40 \mathrm{~nm})$ and $F$ the force applied by the tip. Using this expression of $\sigma$, we have fitted the low force experimental results in 3 with 3 . The agreement between the experiment and the model is satisfactory. The value of $\alpha$ brought by the fit is 130, which gives an order of magnitude of the number of molecules, the elastic state of which is modified by the tip.

The high force regime shows a completely different behavior and can be ascribed to an atomic wear phenomenon. Following the analysis of nano-tribological experiments on calcite in aqueous solution, we consider that the force applied by the tip is high enough to detach ions along the tip-step contact, which means that the tip scratches the step via a mechanical promotion of the kink nucleation. ${ }^{28}$ As this mechanically-favored kink nucleation is thermally activated, the step wear is expected to exhibit an Arrhenius exponential evolution with the force: ${ }^{28}$

$$
v=v_{0} \exp \left(\frac{V_{\mathrm{a}} \sigma}{R T}\right)
$$

with $v_{0}$ a preexponential factor and $V_{\mathrm{a}}$ an activation volume. The fit of the experimental data at high force with this equation is shown in 3 . The agreement is satisfactory and leads to 


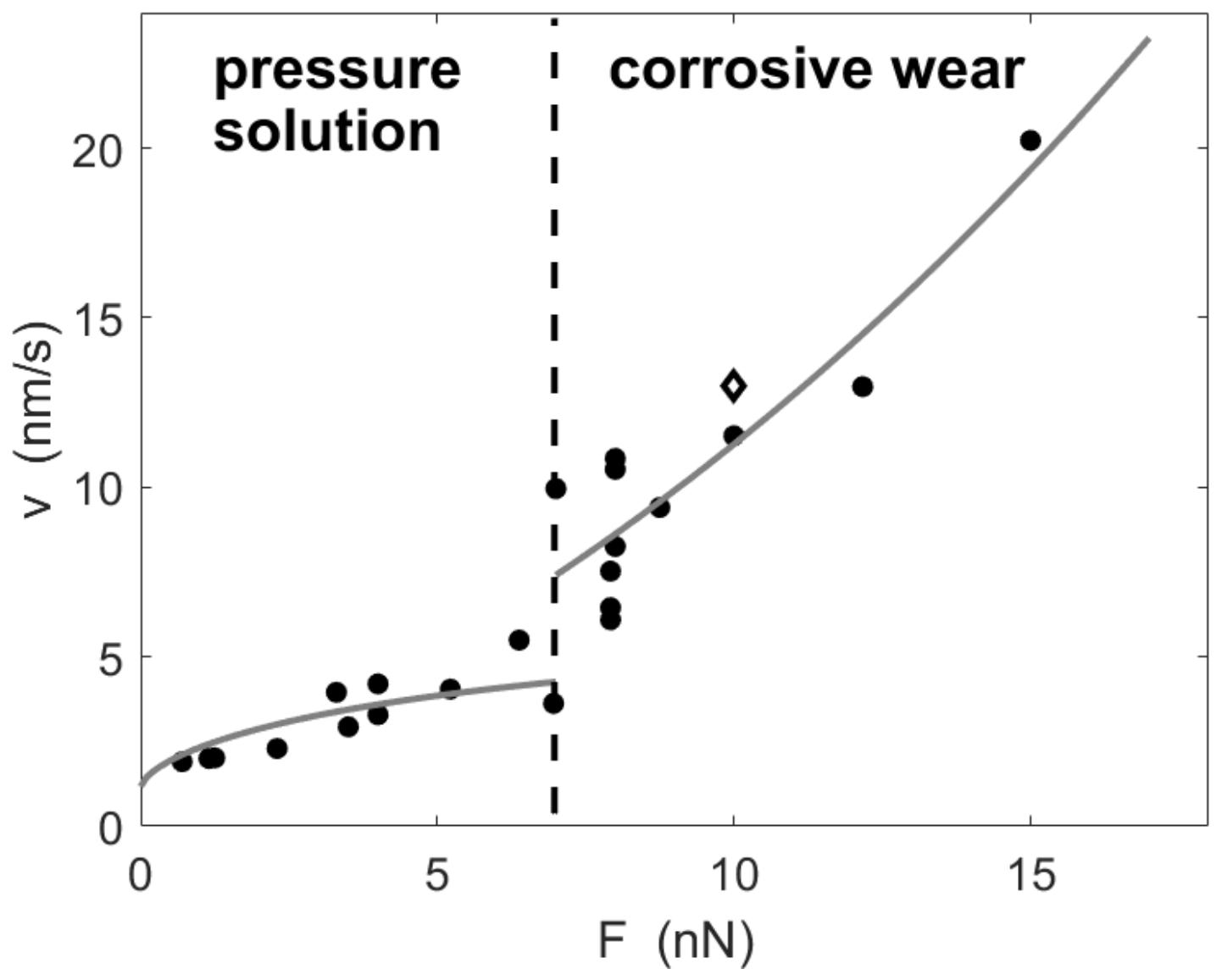

Figure 3: Step velocity in the [100] direction as a function of the force applied by the AFM tip on a gypsum surface for a $90 \%$ saturation index of the flowing solution. The open diamondshaped dot is the datum from Hall \& Cullen. ${ }^{23}$ The low force dots are fitted with 3 and the high forces ones with 4 
confirm that the increase of velocity in this regime is due to a corrosive wear phenomenon in the case of gypsum, as was the case for calcite. ${ }^{28}$ The best-fit value of the activation volume is $V_{\mathrm{a}} \sim 100 \mathrm{~cm}^{3} \cdot \mathrm{mol}^{-1}$. The molar volume of monoclinic gypsum being $74 \mathrm{~cm}^{3} \cdot \mathrm{mol}^{-1}$, this fitted value tends to show that the tip promotes the removal of one or two unit cells.

The existence of this wear regime enables to understand the unexpectedly high value measured by Hall \& Cullen, shown in 2. These authors are the only ones mentioning the force they apply on the surface with the tip: $F=10 \mathrm{nN}$. This value lies clearly in the corrosive wear domain, and the velocity they measure $(v \sim 13 \mathrm{~nm} / \mathrm{s})$ is consistent with the one we measure with this applied force, as can be checked in 3. Hence their experiment investigates tribologically-enhanced dissolution, not spontaneous dissolution, and their result is therefore outside the range of expected values of step velocity during dissolution (see 2).

The force for which the transition between pressure solution and corrosive wear of the fast steps occurs, $F_{\mathrm{f}} \sim 7 \mathrm{nN}$, is very close to the equivalent value for the slow steps, $F_{\mathrm{s}} \sim 10 \mathrm{nN} .{ }^{26}$ It should therefore stem from the same molecular quantity, most probably the binding force of the structural water of the gypsum molecule with the surface.

We can infer from this investigation of the bias induced by the AFM tip on the dissolution process that, to get reliable and reproducible values of the step velocities, a particular care will have to be taken subsequently (i) to choose a small applied force, at least smaller than $7 \mathrm{nN}$, (ii) to keep the value of the force similar for all velocity measurements. These two conditions have been always fulfilled thereafter in performing all experiments with a $1.2 \pm 0.7 \mathrm{nN}$ force.

\section{Dissolution rate from the step velocity}

The heterogeneity of the molecular mechanisms at the basis of dissolution, and the resulting diversity of the local dissolution rates, has been recently acknowledged. ${ }^{15}$ In the case of calcite marble, the local rates measured in a plane surface span from 0.03 to $0.7 \times 10^{-6} \mathrm{~mol} \mathrm{~m}^{-2} \mathrm{~s}^{-1}$, originating from dislocation-driven etch pit growth, vacancy-driven etch pit broadening, etch 
pit coalescence ... ${ }^{14}$ The experimental values of macroscopic rate found in the literature, measured in very diverse samples (single crystals, polycrystals, powders ...), range from 0.3 to $5 \times 10^{-6} \mathrm{~mol} \mathrm{~m}^{-2} \mathrm{~s}^{-1}$ in far from equilibrium conditions. ${ }^{12}$ We note that the order of magnitude of the rates do not agree. This discrepancy may originate from the shape of the samples, grains being likely to be more reactive than plane surfaces, due to higher step and kink density, so to show larger rates. This tiny overlap of the rates of both scales emphasizes the fact that developments in the statistical analysis of the basic mechanisms and their interaction are still needed to recover a unified view of the dissolution of calcite. ${ }^{29}$

Two main strategies exist for accessing the microscopic dissolution rates. The first one uses VSI and measures directly the microscopic rate from the matter loss deduced from the local vertical retreat of the surface. The link between a local rate and its corresponding atomic mechanism is made from the interpretation of the topographic changes. The advantage of this technique is that it provides a map of the microscopic rates (the integration of which gives the overall value), the drawback is that measurements are carried out ex situ (outside the solution). Our study concerns the second one, that uses AFM and attempts to compute microscopic rates from the measured kinetics of atomic events (step velocity, pit spreading ...). The advantage of this method is that the measurements are performed in situ (during dissolution), the drawback is that the computation of the overall rate from the kinetics of microscopic events is not straightforward.

In the case of gypsum, we have noticed from the AFM measurements that etch pits do not seem to play a leading role. Indeed they never get deep or very wide. This absence of noticeable growth is not a consequence of a large density of pits, which would lead the pits to coalesce before having time to grow. This limited growth is a consequence of the sweeping of the surface by the rough steps, which make regularly the pits disappear. From this overall assessment of the dissolution processes, we can make the assumption that the phenomenon is driven by the rough atomic step motion.

We have observed that the migration of three families of steps contributes to the release 
of matter from solid to liquid, the ultimate measure of which is the dissolution rate. To quantitatively estimate the total quantity of matter liberated from the solid by step migration, two quantities are required: the velocity of the steps $v$, and their number density $n$ at the surface. The resulting dissolution rate $R$ is ${ }^{30}$

$$
R=\frac{v n h}{\bar{V}}
$$

with $h$ the height of a step $(h=0.75 \mathrm{~nm})$ and $\bar{V}$ the molar volume of gypsum $(\bar{V}=$ $\left.74 \mathrm{~cm}^{3} \cdot \mathrm{mol}^{-1}\right)$.

The values of $v$ and $n$ for 5 values of the saturation index have been collected in 1 . From them and 5, the dissolution rate resulting from the migration of all 3 steps has been drawn in 4 for the 5 saturation indices. At first glance, we see that the contribution from the fast and slow steps are completely negligible, their velocity being much too small to be comparable with the one of the highly unstable rough steps. Accordingly the displacement of the [u0w] steps is the only contributor to the dissolution rate.

Table 1: Velocity and number density of the slow [001], fast [100] and rough [u0w] steps during the dissolution of the cleavage plane (010) of gypsum in flowing aqueous solutions of calcium sulfate of saturation index $\Omega$.

\begin{tabular}{c|cccccc}
$\Omega(\%)$ & $\begin{array}{c}\mathrm{v}[001] \\
(\mathrm{nm} / \mathrm{s})\end{array}$ & $\begin{array}{c}\mathrm{n}[001] \\
\left(\mu \mathrm{m}^{-1}\right)\end{array}$ & $\begin{array}{c}\mathrm{v}[100] \\
(\mathrm{nm} / \mathrm{s})\end{array}$ & $\begin{array}{c}\mathrm{n}[100] \\
\left(\mu \mathrm{m}^{-1}\right)\end{array}$ & $\begin{array}{c}\mathrm{v}[\mathrm{u} 0 \mathrm{w}] \\
(\mathrm{nm} / \mathrm{s})\end{array}$ & $\begin{array}{c}\mathrm{n}[\mathrm{u} 0 \mathrm{w}] \\
\left(\mu \mathrm{m}^{-1}\right)\end{array}$ \\
\hline 75 & 1.18 & 0.48 & 5.64 & 0.11 & 1900 & 0.54 \\
80 & 0.88 & 0.44 & 5.45 & 0.11 & 2230 & 0.43 \\
85 & 0.56 & 0.24 & 5.28 & 0.10 & 757 & 0.68 \\
90 & 0.60 & 0.10 & 2.42 & 0.08 & 494 & 0.50 \\
94 & 0.58 & 0.10 & 2.62 & 0.07 & 391 & 0.39
\end{tabular}

To compare these values inferred from atomic events with the macroscopic dissolution rates, we have added in 4 the evolution of the macroscopic experimental $R$ with the undersaturation. This $R(\Omega)$ line stems from an analysis of almost all published experimental data of gypsum dissolution (mostly coming from solution chemistry techniques). In these experiments, the solution is always flowing and thereby the measured dissolution rate contains 


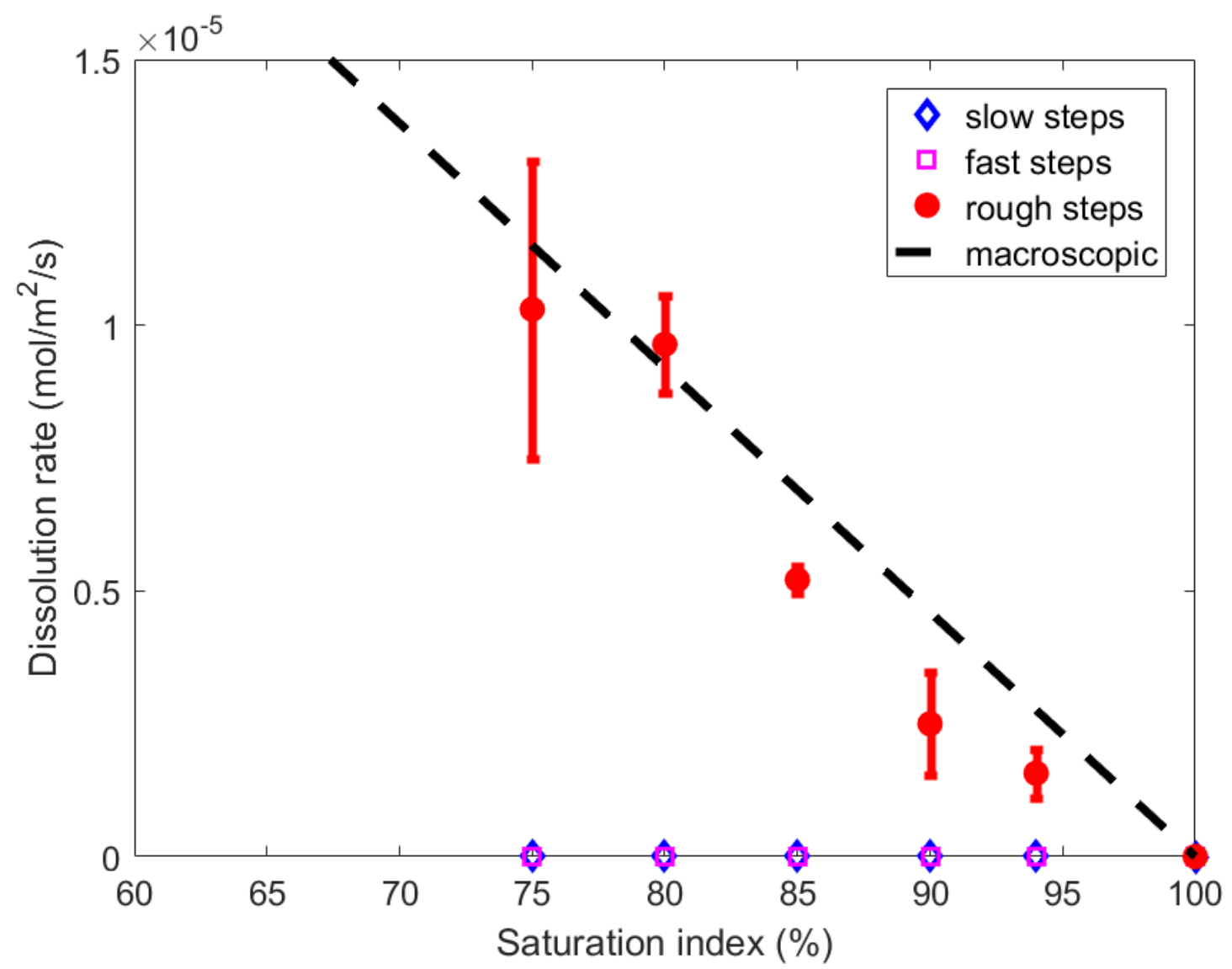

Figure 4: Dissolution rates resulting from the fast, slow and rough steps motion at the surface of a gypsum single crystal for various saturation indices of the flowing solution. The dashed line is the macroscopic pure dissolution rate of gypsum, compatible with all published experimental values ${ }^{25}$. 
also a contribution from convection, varying with the stirring rate, dissolving particle size ... This results in highly dispersed and inconsistent values. Once the blurring influence of hydrodynamics has been removed, one well-defined dissolution rate constant can be drawn from all the experiments. ${ }^{25}$ This rate constant has been validated by an original measurement using holographic interferometry in a non-flowing solution, thereby free from any hydrodynamical contribution. ${ }^{31}$ Consequently the $R(\Omega)$ line in 4 represents (i) pure dissolution rate values, (ii) compatible with all the published values of $R$.

4 shows that the agreement between the atomic and macroscopic dissolution rate is extremely good. We stress on the fact that both measurements are completely independent, no adjustment has been performed. The fact that both agree quantitatively is a proof that the dissolution of gypsum is completely driven by one well-defined atomic mechanism, namely the migration of $[\mathrm{u} 0 \mathrm{w}]$ steps.

This particular analysis, dedicated to gypsum, is the first one showing a perfect agreement between measurements performed at the two extreme scales of dissolution. It shows that a thorough examination of the molecular contributions to the overall dissolution permits to bridge the gap quantitatively between the nanometric and macroscopic values. Obviously, this examination has to be completed for every different mineral, and may sometimes be less direct than in the case of gypsum, but it emphasizes the predictive power of AFM experiments.

\section{Conclusion}

Dissolution rates deduced from atomic step velocity or etch pit growth are hardly ever consistent with rates measured macroscopically. We measure here with atomic force microscopy dissolution rates deduced from atomic motions at the surface of gypsum in complete agreement with macroscopic rates. This consistency can be obtained provided that the bias induced by the promotion of dissolution by the stress applied by the tip is taken into ac- 
count, and that all atomic mechanisms occurring during dissolution are identified and the driving one ascertained.

In showing that a coherence can be reached between usually incompatible scales of dissolution, we do not mean that the deduction of macroscopic rates from atomic mechanisms is straightforward. In other minerals, like carbonates or alumino-silicates, it is possible that no unique driving molecular process exists, and that the interaction between several ones has to be understood and modeled, for instance etch pits merging rate and vertical surface retreat. In this case, a concept like the dissolution rate spectrum reveals necessary and provides a satisfactory description of the heterogeneity of the surface reactivity, i.e., of the spatial distribution of atomic mechanisms. ${ }^{15}$ It has also recently been shown that the access to the rate spectrum of one particular polycrystalline material with VSI enables to identify the main defects acting as source of etch pits during dissolution, which in turn can be used to calibrate a kinetic Monte Carlo simulation code, thus improving its predictive power. ${ }^{14}$

But we have shown here that, if AFM does not give a direct access to dissolution rates, unlike VSI, it enables a crucial real-time observation of the atomic processes of the phenomenon.

\section{Acknowledgements}

This work was supported by the European Training Network Nanoheal. Nanoheal has received funding from the European Union's Horizon 2020 research and innovation program under the Marie Sklodowska-Curie grant agreement No 642976. The results of this paper reflects only the authors view and the European Commission is not responsible for any use that may be made of the information it contains. 


\section{References}

(1) Lüttge, A.; Winkler, U.; Lasaga, A. Interferometric study of the dolomite dissolution: a new conceptual model for mineral dissolution. Geochim. Cosmochim. Acta 2003, 67, 1099.

(2) Lasaga, A.; Lüttge, A. Variation of crystal dissolution rate based on a dissolution stepwave model. Science 2001, 291, 2400.

(3) Teng, H. Controls by saturation state on etch pit formation during calcite dissolution. Geochim. Cosmochim. Acta 2004, 68, 253-262.

(4) Arvidson, R.; Lüttge, A. Mineral dissolution kinetics as a function of distance from equilibrium âĂŞ New experimental results. Chem. Geol. 2010, 269, 79.

(5) Jeschke, A.; Vosbeck, K.; Dreybrodt, W. Surface controlled dissolution rates of gypsum in aqueous solutions exhibit nonlinear dissolution kinetics. Geochim. Cosmochim. Acta 2001, 65, 27.

(6) Raines, M.; Dewers, T. Mixed transport / reaction control of gypsum dissolution kinetics in aqueous solutions and initiation of gypsum karst. Chem. Geol. 1997, 140, 29.

(7) Herrero, J.; Artieda, O.; Hudnall, W. Gypsum, a tricky material. SSSAJ 2009, 73, 1757-1763.

(8) Pachon-Rodriguez, E.; Guillon, E.; Houvenaghel, G.; Colombani, J. Wet creep of hardened hydraulic cements âĂ $\breve{T}$ Example of gypsum plaster and implication for hydrated Portland cement. Cem. Concr. Res. 2014, 63, 67-74.

(9) Wolthers, M. How minerals dissolve. Science 2015, 349, 1288. 
(10) Shiraki, R.; Rock, P.; Casey, W. Dissolution kinetics of calcite in $0.1 \mathrm{M} \mathrm{NaCl}$ solution at room temperature: an atomic force microscopic (AFM) study. Aquat. Geochem. 2000, 6, 87-108.

(11) Duckworth, O.; Martin, S. Dissolution rates and pit morphologies of rhombohedral carbonate minerals. Am. Mineral. 2004, 89, 554-563.

(12) Colombani, J. The alkaline dissolution rate of calcite. J. Phys. Chem. Lett. 2016, 7, $2376-2380$.

(13) Kurganskaya, I.; Lüttge, A. Kinetic Monte Carlo approach to study carbonate dissolution. J. Phys. Chem. C 2016, 120, 6482-6492.

(14) Fischer, C.; Lüttge, A. Beyond the conventional understanding of waterâ ĂŞrock reactivity. Earth Planet. Sci. Lett. 2017, 45\%, 100-105.

(15) Fischer, C.; Arvidson, R.; Lüttge, A. How Predictable are Dissolution Rates of Crystalline Material? Geochim. Cosmochim. Acta 2012, 98, 177-185.

(16) Shindo, H.; Kaise, M.; Kondoh, H.; Nishihara, C.; Hayakawa, H.; Ono, S.; Nozoye, H. Structure of cleaved surfaces of gypsum studied with atomic force microscopy. J. Chem. Soc. Chem. Commun. 1991, 16, 1097.

(17) Pachon-Rodriguez, E.; Colombani, J. Pure dissolution kinetics of anhydrite and gypsum in inhibiting aqueous salt solutions. AIChE J. 2012, 59, 1622.

(18) Gasperino, D.; Yeckel, A.; Olmsted, B.; Ward, M.; Derby, J. Mass transfer limitations at Crystallizing interfaces in an atomic force microscopy fluid cell: A finite element analysis. Langmuir 2006, 22, 6578-6586.

(19) Peruffo, M.; Mbogoro, M.; Adobes-Vidal, M.; Unwin, P. Importance of mass transport and spatially heterogeneous flux processes for in situ atomic force microscopy mea- 
surements of crystal growth and dissolution kinetics. J. Phys. Chem. C 2016, 120, $12100-12112$.

(20) Burgos-Cara, A.; Putnis, C.; Rodriguez-Navarro, C.; Ruiz-Agudo, E. Hydration effects on gypsum dissolution revealed by in situ nanoscale atomic force microscopy observations. Geochim. Cosmochim. Acta 2016, 179, 110-122.

(21) Fan, C.; Teng, H. Surface behavior of gypsum during dissolution. Chem. Geol. 2007, $245,242$.

(22) Bosbach, D.; Rammensee, W. In situ investigation of growth and dissolution on the (010) surface of gypsum by Scanning Force Microscopy. Geochim. Cosmochim. Acta 1994, 58, 843 .

(23) Hall, C.; Cullen, D. Scanning Force Microscopy of gypsum dissolution and crystal growth. AIChE J. 1996, 42, 232.

(24) Peruffo, M.; Mbogoro, M.; Edwards, M.; Unwin, P. Holistic approach to dissolution kinetics: linking direction-specific microscopic fluxes, local mass transport effects and global macroscopic rates from gypsum etch pit analysis. Phys. Chem. Chem. Phys. 2013, 15, 1956-1965.

(25) Colombani, J. Measurement of the pure dissolution rate constant of a mineral in water. Geochim. Cosmochim. Acta 2008, 72, 5634.

(26) Pachon-Rodriguez, E.; Piednoir, A.; Colombani, J. Pressure solution at the molecular scale. Phys. Rev. Lett. 2011, 10\%, 146102.

(27) Gratier, J.; Dysthe, D.; Renard, F. The role of pressure solution creep in the ductility of the earth's upper crust. Adv. Geophys. 2013, 54, 47-179.

(28) Park, N.; Kim, M.; Langford, S.; Dickinson, J. Atomic layer wear of single-crystal calcite in aqueous solution scanning force microscopy. J. Applied Phys. 1996, 80, 2680. 
(29) Morse, J.; Arvidson, R. The dissolution kinetics of major sedimentary carbonate minerals. Earth-Sci. Rev. 2002, 58, 51-84.

(30) Vinson, M.; Lüttge, A. Multiple length-scale kinetics: an integrated study of calcite dissolution rates and strontium inhibition. Am. J. Sci. 2005, 305, 119-146.

(31) Colombani, J.; Bert, J. Holographic interferometry study of the dissolution and diffusion of gypsum in water. Geochim. Cosmochim. Acta 2007, 71, 1913. 


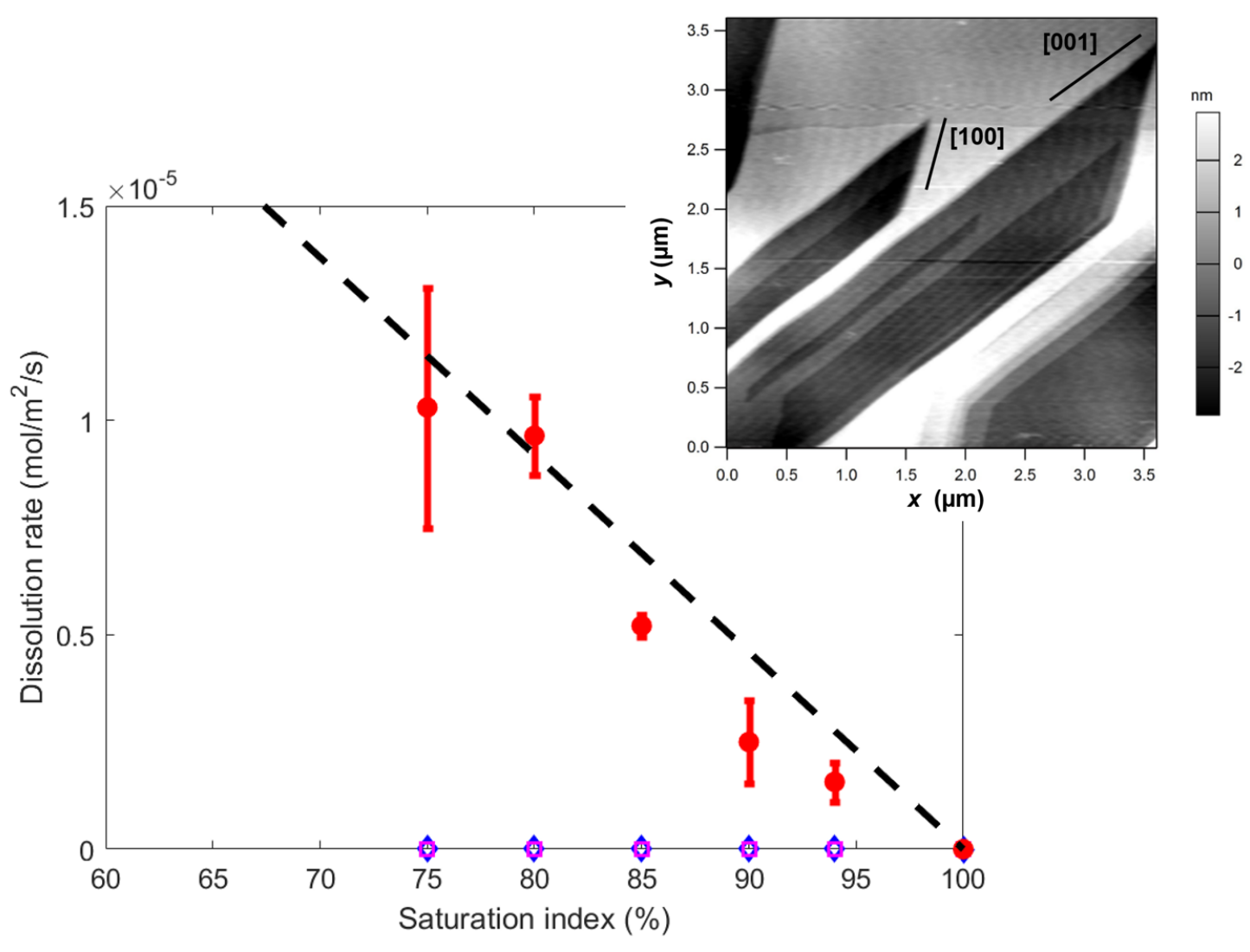

TOC graphic 